\title{
Cardiología para pediatras de Atención Primaria
}

\author{
J. Ayala Curiel \\ Servicio de Cardiología Pediátrica. Departamento de Pediatría, \\ Hospital de Cruces. Barakaldo, Vizcaya. España.
}

Rev Pediatr Aten Primaria. 2009; I I (Supl I 7):s45 I-s456

Javier Ayala Curiel, javier.ayalacuriel@osakidetza.net

\section{Resumen}

La Cardiología infantil es la especialidad pediátrica que se encarga de la asistencia integral al niño (desde la concepción hasta la adolescencia) con enfermedad congénita o adquirida del sistema cardiovascular. Aunque la herramienta principal para el diagnóstico es la ecocardiografía, la anamnesis y la exploración física son fundamentales para sospechar una cardiopatía, de ahí que el pediatra de Atención Primaria tenga en este campo una labor crucial. Con entrenamiento, este será capaz de detectar con una alta fiabilidad una cardiopatía mediante la auscultación.

Palabras clave: Cardiología pediátrica, Auscultación, Soplos cardíacos, Cardiopatía congénita.

\section{Abstract}

Paediatric cardiology is the speciality that refers to the care of children (from conception through adolescence) with congenital or acquired disorders of the heart and vessels. Although the main diagnostic tool is echocardiography, medical history and physical examination are essential to suspect heart diseases, hence the primary care paediatrician in this field has crucial work. With training, they will be able to detect, with high reliability, heart disease by auscultation.

Key words: Paediatric cardiology, Auscultation, Heart murmurs, Congenital heart disease.

\section{Introducción}

La Cardiología infantil es la rama de la Pediatría que se ocupa del niño con enfermedad congénita o adquirida del sistema cardiovascular. Como iremos viendo, el elevado número de niños con cardiopatías o con síntomas relacionados con el corazón, hace que el papel del pediatra de Atención Primaria tenga una importancia crucial para su diagnóstico y manejo. El objetivo de este taller es aprender a diagnosticar a un niño con cardiopatía mediante la anamnesis y la exploración física.

\section{Taller}

El gran desarrollo tecnológico y la alta subespecialización que ha experimenta-

El autor declara no presentar posibles conflictos de intereses en relación con la preparación y publicación de este artículo. 
do la Cardiología pediátrica en los últimos años, con herramientas tan lejanas para el pediatra de Atención Primaria, y casi impronunciables para cualquiera, como la tomografía computadorizada (TC) helicoidal, la ecocardiografía fetal o el speckel tracking, puede llevar a aquel a preguntarse: si es todo tan técnico, tan específico, ¿por qué tener conocimientos de cardiología?, ¿y para qué? La respuesta es clara y merece el esfuerzo que supone entrenarse en el diagnóstico y manejo de un niño con cardiopatía ${ }^{1-3}$ :

- Las cardiopatías son la malformación congénita más frecuente, de modo que aproximadamente uno de cada 100 recién nacidos que un pediatra recibe en su cupo tendrá una cardiopatía. De estos, casi la mitad necesitará cirugía.

- Causan el 35\% de los fallecimientos por anomalía congénita en niños y el $10 \%$ de todos los fallecimientos durante el primer año de vida.

- En ciertas cardiopatías hay un riesgo de sufrir eventos fatales.

Por todo esto, el objetivo principal del estudio de un niño con sospecha de cardiopatía es el diagnóstico precoz. Las herramientas fundamentales para iniciar dicho estudio son la anamnesis y la exploración física. La anamnesis se basa en las preguntas clásicas: ¿qué le pasa al niño?, ¿desde cuándo?, ¿a qué lo atribuye la familia?

Los síntomas con los que un niño con cardiopatía puede presentarse en la consulta de su pediatra son variados, pero habrá que estar alerta, sobre todo, ante un niño con cansancio y sudoración con el ejercicio, sabiendo que un lactante lo realiza durante la alimentación. Ante un niño con fallo de medro, asociado o no a episodios frecuentes de infecciones de vías respiratorias, es obligado incluir en el diagnóstico diferencial las cardiopatías congénitas. Otros síntomas frecuentes de consulta, pero con menor incidencia de cardiopatía, son el dolor torácico, los síncopes o mareos y las palpitaciones.

Los antecedentes, tanto personales como familiares, pueden ayudarnos al diagnóstico. En cuanto a los personales, hay que comenzar por los obstétricos, preguntando por enfermedades maternas durante el embarazo, sobre todo diabetes mellitus y lupus eritematoso sistémico, o toma de ciertos fármacos como anticonvulsivantes o litio. Los recién nacidos con cardiopatía congénita tienen estadísticamente un peso inferior al que tienen los niños sanos, y es este un dato a tener en cuenta, aun siendo muy poco especíicico. Como es sabido, algunas enfermedades genéticas se asocian con fre- 
cuencia a cardiopatías y las más habituales que pueden verse en el centro de salud son los síndromes de Down, Turner, Noonan, Williams y Duchenne. Hay que investigar antecedentes familiares de cardiopatías congénitas, síncopes, muerte súbita en personas jóvenes o enfermedades del tejido conectivo como el Marfan, y arritmias como la enfermedad de Wolff-Parkinson-White o el síndrome QT largo.

Pero siendo esto importante, es imprescindible saber que muchos niños con cardiopatía carecen de antecedentes familiares u obstétricos de interés que nos den alguna pista, su historia personal es normal, no se presentan en la consulta con ningún síntoma y están aparentemente sanos. Ante un escenario tal, el pediatra se preguntará: ¿cómo sabré, entonces, que este niño tiene una cardiopatía? La respuesta brilla nuevamente y es la base de este taller: este niño tiene una exploración patológica. ¿Qué datos son, entonces, cruciales en la exploración física de un niño con cardiopatía congénita? Hay que fijarse en el fenotipo, por lo anteriormente expuesto, en signos de dificultad respiratoria (polipnea y tiraje) y en el color de la piel en busca de cianosis o subcianosis. Se debe palpar el mesocardio y el hueco supraesternal para detectar un frémito o thrill, tocar los pulsos proximales y distales y descartar la presencia de hepatomegalia. La auscultación cardíaca debe hacerse de forma reglada, en los cuatro focos principales, en el hemitórax derecho y en la espalda. La exploración física y su correspondiente cardiopatía pueden diferir según las distintas etapas de la vida, por lo cual veremos los datos exploratorios en los que hay que fijarse según la edad del niño:

- Es muy importante realizar la primera exploración de un recién nacido en el centro de salud hacia la primera semana de vida, pues algunas cardiopatías potencialmente graves (como la coartación de aorta) pueden pasar desapercibidas en los primeros días. Así, se debe poner especial atención en la auscultación de la espalda (buscando un soplo) y en la palpación de los pulsos. Otras cardiopatías que suelen debutar en período neonatal o de lactante pequeño son los cortocircuitos, y en especial la comunicación interventricular, con dificultades para la alimentación, fallo de medro, fatiga y soplo cardíaco.

- El lactante con cardiopatía congénita habitualmente es un niño sano que presenta un soplo, pero también hay que valorar cuidadosa- 
mente los signos de dificultad respiratoria $y$, en concreto, los episodios de bronquiolitis que pueden estar enmascarando una enfermedad del corazón con hiperaflujo pulmonar, como una comunicación interventricular, un canal auriculoventricular o una persistencia del conducto arterioso. Como hemos dicho antes, un fallo de medro debe alertarnos de la posibilidad de encontrarnos ante una cardiopatía.

- En el niño escolar y el adolescente puede detectarse una cardiopatía a partir de un soplo no escuchado anteriormente, como ocurre con las comunicaciones interauriculares, 0 de "ruidos" cardíacos anormales (el clic de eyección de una aorta bicúspide). También es esta una época en la que se debe controlar la tensión arterial para diagnosticar una coartación aórtica que haya podido pasar desapercibida.

Aun tratándose este de un taller eminentemente práctico, intentaremos aquí definir cómo es una auscultación normal y patológica (en sus formas más frecuentes) en la edad pediátrica. Esta diferenciación es crucial para la correcta derivación, por parte del pediatra de Atención Primaria, a una consulta de Cardiología infantil. Esta derivación se hará en mu- chas ocasiones sospechando una cardiopatía concreta.

Primero, hay que escuchar el primer y el segundo ruido, y con ellos delimitar la sístole y la diástole, y medir la frecuencia cardíaca. Hay características del ciclo cardíaco que no son patológicas, y las más habituales son la arritmia respiratoria y el desdoblamiento fisiológico del segundo ruido, que tienen igual base fisiológica. Es muy importante conocer las características de un soplo inocente y distinguirlo de uno patológico, y la única manera de alcanzar tal momento decisivo es utilizar el estetoscopio con asiduidad y comparar lo que oímos con el diagnóstico final que el cardiólogo pediátrico emita.

Un soplo inocente tiene un sonido muy característico. Es piante o vibratorio, de bajo grado, se escucha en el mesocardio y el ápex, al inicio de la sístole, y se acompaña de un segundo ruido normal. Disminuye con la sedestación y aumenta con la espiración y los estados hiperdinámicos como la fiebre. Un tipo muy frecuente de soplo inocente es el soplo venoso. Aparece generalmente entre los 3-6 años y está producido por el paso de la sangre en las venas del cuello, por lo que se escucha en la región infraclavicular, con el niño sentado, como un soplo continuo que se irradia hacia los focos de la base. Disminuye típicamente con la la- 
teralización contralateral de la cabeza y el decúbito, y desaparece al comprimir la vena yugular.

Hay datos auscultatorios que pueden deberse a una cardiopatía. En estos casos hay que estar muy alerta para sospecharla. Son los siguientes:

- Trastornos de la frecuencia cardíaca, con taquicardia o bradicardia.

- Desdoblamiento fijo del segundo ruido, que suele acompañar a las comunicaciones interauriculares grandes.

- Clic de eyección en ápex que, aunque en ocasiones es normal, también es típico de la aorta bicúspide.

- Ritmo de galope, que aparece en casos de insuficiencia cardíaca.

- Soplos patológicos, de los que vamos a definir los más usuales. Hay que pensar que nos encontramos ante un soplo patológico cuando este sea pansistólico o diastólico, de carácter eyectivo o rudo y de alto grado, y cuando se acompañe de frémito o un segundo ruido desdoblado. El soplo de comunicación interventricular es tan característico como frecuente y se escucha en el borde esternal izquierdo, irradiándose en banda. El de estenosis pulmonar es rudo, en el foco pulmonar y en casos severos se irradia a todos los focos, las axilas y la espalda. La estenosis aórtica provoca un soplo igualmente rudo pero que es máximo en el foco aórtico y suele acompañarse de un frémito en el hueco supraesternal. La persistencia del conducto arterioso da lugar a un soplo continuo, suave, en el foco pulmonar, palpándose unos pulsos saltones. La comunicación interauricular tiene un soplo inespecífico, pero sospecharemos esta cardiopatía cuando se acompañe, como ya hemos dicho, de un desdoblamiento fijo del segundo ruido. Llegados a este punto del taller, en el cual somos capaces de decir, mediante la anamnesis y la exploración, que un niño tiene probablemente una cardiopatía, y en ocasiones qué cardiopatía, es lógico que el asistente al mismo se haga dos preguntas:

- ¿Qué prueba complementaria realizo?

- ¿Cuándo derivo a Cardiología pediátrica a un niño?

En cuanto a la prueba complementaria, hay que saber que la radiografía de tórax no solo no ayuda al diagnóstico sino que puede confundirnos, y que el electrocardiograma no nos ofrece ninguna ventaja si el soplo que escuchamos es inocente. Indudablemente, la herramienta más im- 
portante para hacer el diagnóstico preciso de una cardiopatía es la ecocardiografía, con lo que hemos de volver al inicio de este artículo para dar definitiva respuesta a la primera pregunta: el pediatra de Atención Primaria debe ser capaz de sospechar una cardiopatía mediante la anamnesis y la exploración física.

Las indicaciones de derivación se deben individualizar pero, en general, se harán ante:

- La sospecha de un soplo patológico.

- Si un niño tiene un soplo y menos de 1-2 años.

- Si hemos hecho un electrocardiograma y este es patológico.

- Si hay ansiedad familiar, puesto que el corazón es uno de los órganos que más angustia causa.

- Si tenemos dudas diagnósticas, siendo aquí básico que haya una relación fluida entre el pediatra de
Atención Primaria y el cardiólogo para poder hacer feedback, es decir, aprender de la experiencia.

\section{Conclusiones}

A pesar de que la Cardiología pediátrica es una especialidad en la cual la tecnología ha llegado a convertirse en una herramienta indispensable para el quehacer diario, la sospecha inicial que nos lleva a usar esta técnica continúa haciéndose a partir de la anamnesis y la exploración física. Y es aquí, lejos de ecógrafos y cateterismos, donde la labor del pediatra de Atención Primaria se convierte en fundamental. Este taller pretende, humildemente, contribuir a que estos profesionales continúen sintiendo la necesidad de conocer los aspectos básicos de la Cardiología pediátrica para que, de este modo, los niños con cardiopatía reciban una asistencia de calidad desde el inicio. Ellos y sus familias merecen el esfuerzo.

\section{Bibliografía}

1. Aguas Benito F. Valoración y conducta a seguir ante el hallazgo de un soplo cardíaco. En: Muñoz Calvo MT, ed. Pediatría extrahospitalaria. Manual de diagnóstico y tratamiento. 2. ${ }^{a}$ ed. Madrid: Díaz de Santos, S.A.; 1994. p. 183-7.
2. Yi M, Kimbal T, Tsevat J. Evaluation of heart murmurs in children: cost-effectiveness and practical implications. J Pediatr. 2002;141:504-11.

3. Gardiner $S$. Are routine chest $X$ ray and ECG examinations helpful in the evaluation of asymptomatic heart murmurs? Arch Dis Child. 2003;88: 638-40. 